\title{
The traveling wave solution of $(3+1)$ dimensional Kadomtsev-Petviashvili equation by using the firstintegral method
}

\author{
Chunhuan Xiang ${ }^{1, a}$, Honglei Wang ${ }^{2, b^{*}}$
}

${ }^{1}$ School of Public Health and Management, Chongqing Medical University, Chongqing, P.R. China

${ }^{2}$ College of medical informatics, Chongqing Medical University, Chongqing 400016, P.R. China

aw8259300@126.com, ${ }^{b}$ w8259300@163.com, "Corresponding author

Keywords: Kadomtsev-Petviashvili equation, traveling wave solution, the firstintegral method, nonlinear partial differential equations

Abstract. The first integral method, based on the theory of commutative algebra, is an efficient method for obtaining traveling wave solutions of some nonlinear partial differential equations, which is applied to solve $(3+1)$ dimensional Kadomtsev-Petviashvili equation in this work. The traveling wave solutions for the equation are obtained. This method can be applied to nonintegrable equations as well as to integrable ones.

\section{Introduction}

During the past three decades, nonlinear partial differential equations, which are widely used to describe complex phenomena in various fields of science, had recently proved to be valuable tools to the modeling of many physical phenomena, and have gained the focus of many studies due to their frequent appearance in various applications such as fluid flow, signal processing, control theory, systems identification, finance, fractional dynamics, and other areas. Many powerful methods for obtaining traveling wave solutions of nonlinear partial differential equation have been presented, hyperbolic function method[1], F-expansion method[2], Jacobi elliptic function expansion method[3], Riccati equation method[4], tanh-sech method[5, 6, 7]. The first integral method was first proposed for solving Burger-KdV equation [8,9] which is based on the ring theory of commutative algebra[10]. The useful first integral method is widely used by many such as in $[11,12,13,14,15,16,17,18]$ and by the reference therein. The $(3+1)$ dimensional Kadomtsev-Petviashvili equation is studied in this work, which was first written in 1970 by Soviet physicists Boris B. Kadomtsev and Vladimir I. Petviashvili. The equation, came as a natural generalization of the $\mathrm{KdV}$ equation, can be used to model water waves of long wavelength with weakly non-linear restoring forces and frequency dispersion and can also be used to model waves in ferromagnetic media, as well as two-dimensional matter-wave pulses in BoseEinstein condensates[19].

\section{The first-integral method}

For a given nonlinear partial differential equation in the form

$$
P(u, u t, u x, u t t, u x x, u x t, u x x x, \ldots)=0,
$$

Using a wave variable $\xi=\mathrm{k}(\mathrm{x}+\mathrm{ly}+\mathrm{pz}-\lambda \mathrm{t})$, Eq.(1) is changed into ordinary differential equation, which can be rewritten as

$$
Q\left(u, u^{\prime}, u^{\prime \prime}, u^{\prime \prime \prime}, \ldots\right)=0,
$$

The prime in Eq.(2) denotes the derivative with respect to the same variable $\xi$. The equation $f(\xi)=$ $u(x, t)$ is supposed to be the solutions of Eq.(2). Then, a new independent variable is introduced as the following

$$
X(\xi)=f(\xi) ; \quad Y=f \xi(\xi),
$$

which leads a system of nonlinear ordinary differential equations

$$
Y=X \xi(\xi) ; \quad Y \xi=F(X(\xi) ; Y(\xi))
$$


(4)

By employing the qualitative theory of ordinary differential equations [10]. With the same conditions, the general solutions to Eq.(4) can be obtained directly if we can find the integrals for Eq. (4). Generally, it is really difficult for us to realize this result even for the first integral, for a given plane autonomous system, there is no systematic theory that can tell us how to find its first integrals. The Division Theorem can help us obtain one first integral for Eq.(4) which reduces Eq. (2) to a first-order integrable ordinary differential equation. The traveling wave solution for Eq.(1) is obtained by solving the first-order integrable ordinary differential equation. The Division theorem for two variables in the complex domain $\mathbf{C}$ is read as:

Division theorem: Suppose that $G 1[w ; z], G[w ; z]$ are polynomials in $C[w ; z]$ and $G 1[w ; z]$ is irreducible in $C[w ; z]$. If $G[w ; z]$ vanishes at all zero points of $G 1[w ; z]$, then there exists a polynomial $G 2[w ; z]$ in $C[w ; z]$ such that

$$
G[w ; z]=G 1[w ; z] G 2[w ; z]
$$

\section{The (3+1) dimensional Kadomtsev-Petviashvili equation}

The $(3+1)$ dimensional Kadomtsev-Petviashvili equation[20, 21, 22] is studied in this section:

$$
\left(u_{t}+6 u u_{x}+u_{x x x}\right)_{x} \pm 3 u_{y y} \pm 3 u_{z z}=0
$$

By using the transformation $\xi=\mathrm{k}(\mathrm{x}+\mathrm{ly}+\mathrm{pz}-\lambda \mathrm{t})$, where $\mathrm{k}, \mathrm{l}, \mathrm{p}$ and $\lambda$ are constants, and integrating the Eq. (6) with respect to $\xi$ and taking the integration constant as $\mathrm{R}$ yields the ordinary differential equation

Using Eq.(3), we obtain

$$
u_{\xi \xi}+\frac{3 u^{2}}{k^{2}}+\frac{-\lambda \pm 3 l^{2} \pm 3 p^{2}}{k^{2}} u-\frac{R}{k^{2}}=0
$$

$$
\dot{X}=Y ; \quad \dot{Y}=\frac{R}{k^{2}}-\frac{3 u^{2}}{k^{2}}-\frac{-\lambda \pm 3 l^{2} \pm 3 p^{2}}{k^{2}} u ;
$$

According to the first-integral method, $\mathrm{X}(\xi)$ and $\mathrm{Y}(\xi)$ are supposed to be the nontrivial solutions of Eq. (8), we obtain ${ }^{G(X, Y)=\sum_{i=0}^{m} a_{i}(X) Y^{i}=0}$ which is an irreducible polynomial in the complex domain C[X; Y], then the above equation is rewritten as

$$
G(X(\xi), Y(\xi))=\sum_{i=0}^{m} a_{i}(X(\xi)) Y^{i}(\xi)=0
$$

where $a \mathrm{i}(\mathrm{X}(\xi)),(\mathrm{i}=0 ; 1 ; 2 ;::: \mathrm{m})$ are polynomials of $\mathrm{X}$ and $\operatorname{am}(\mathrm{X}(\xi)) \neq 0$. Eq.(9) is called the first integral for Eq.(8). According to the Division Theorem, a polynomial $g(X)+h(X) Y$ in the complex domain $\mathrm{C}[\mathrm{X} ; \mathrm{Y}]$ must meet the relationship

$$
\frac{d G}{d \xi}=\frac{d G}{d X} \frac{d X}{d \xi}+\frac{d G}{d Y} \frac{d Y}{d \xi}=(g(X)+h(X) Y) \sum_{i=0}^{m} a_{i}(X) Y^{i}
$$

Here, we assume that two different cases, $m=1$ and $m=2$ in Eq.(9) in this example.

Case I. Equating the coefficients of $\mathrm{Yi},(\mathrm{i}=0,1,2)$ on both side of Eq.(10) with $\mathrm{m}=1$, we obtain

$$
\begin{aligned}
& a_{1}(X)=h(X) a_{1}(X) \\
& a_{0}(X)=g(X) a_{1}(X)+h(X) a_{0}(X) \\
& g(X) a_{0}(X)=a_{1}(X)\left(\frac{R}{k^{2}}-\frac{3 X^{2}}{k^{2}}-\frac{-\lambda \pm 3 l^{2} \pm 3 p^{2}}{k^{2}} X\right) ;
\end{aligned}
$$

As known $a \mathrm{i}(\mathrm{X})(\mathrm{i}=0 ; 1)$ are polynomials, then $a 1(\mathrm{X})$ is constant and $\mathrm{h}(\mathrm{X})=0$ are obtained from Eq.(11). Usually, $a 1(\mathrm{X})=1$. Balancing the degrees of $\mathrm{g}(\mathrm{X})$ and $a 0(\mathrm{X})$, we conclude that $\operatorname{deg}(\mathrm{g}(\mathrm{X}))=$ 1 only.

$$
a_{0}=\frac{1}{2} A_{1} X^{2}+A_{0} X+B_{0}
$$

is obtained with the condition $\mathrm{g}(\mathrm{X})=\mathrm{A} 0+\mathrm{A} 1 \mathrm{X}$, where $\mathrm{B} 0$ is some an integration constant.

Substituting $a 1$, g, $a 0$, into Eq.(13) and setting all the coefficients of powers $\mathrm{X}$ to be zero, then we obtain a system of nonlinear algebraic equations and by solving it, we obtain

$$
A_{1}=0 ; \quad A_{0}= \pm \frac{\sqrt{\lambda \mp 3 l^{2} \mp 3 p^{2}}}{k} ; \quad B_{0}=\frac{R}{k^{2} A_{0}}
$$


where $\mathrm{k} \neq 0, \lambda, 1$, and $\mathrm{p}$ are constants. Using the conditions Eq.(15) and Eq.(8), we obtain the traveling wave solution of the Eq.(6)

$$
u(x, y, z, t)=-\frac{R}{A_{0}^{2} k^{2}}+C_{0} e^{-A_{0} k(x+l y+p z-\lambda t)}
$$

where $\mathrm{C} 0$ is a constant. obtain

Case II. Equating the coefficients of Yi,(i = 0, 1, 2, 3) on both side of Eq.(10) with $\mathrm{m}=2$, we

$$
\begin{aligned}
& a_{2} \dot{(X)}=h(X) a_{2}(X) \\
& \left.a_{1} \dot{(} X\right)=h(X) a_{1}(X)+g(X) a_{2}(X) \\
& a_{0} \dot{(X)}=g(X) a_{1}(X)+h(X) a_{0}(X)-2 a_{2}\left(\frac{R}{k^{2}}-\frac{3 X^{2}}{k^{2}}-\frac{-\lambda \pm 3 l^{2} \pm 3 p^{2}}{k^{2}} X\right) \\
& g(X) a_{0}(X)=a_{1}(X)\left(\frac{R}{k^{2}}-\frac{3 X^{2}}{k^{2}}-\frac{-\lambda \pm 3 l^{2} \pm 3 p^{2}}{k^{2}} X\right) ;
\end{aligned}
$$

Since $a \mathrm{i}(\mathrm{X})(\mathrm{i}=0,1,2)$ are polynomials, $a 2(\mathrm{X})$ is constant and $\mathrm{h}(\mathrm{X})=0$ are yielded from Eq.(17), we set $a 2=1$ as usual. Balancing the degrees of $\mathrm{g}(\mathrm{X}), a 1(\mathrm{X})$ and $a 2(\mathrm{X})$, we conclude that $\operatorname{deg}(\mathrm{g}(\mathrm{X}))=$ 0 , therefore we have: $\mathrm{g}(\mathrm{X})=\mathrm{A} 1$ is given. $a 1(\mathrm{X})$ is obtained from Eq.(18) as follow

where $\mathrm{A} 0$ is an integration constant.

$$
a 1(\mathrm{X})=\mathrm{A} 1 \mathrm{X}+\mathrm{A} 0
$$

$$
a_{0}(X)=\frac{A_{1}^{2} X^{2}+2 A_{1} A_{0} X}{2}-\frac{2 R X-2 X^{3}+\left(\lambda \mp 3 l^{2} \mp 3 p^{2}\right) X^{2}}{k^{2}} ;
$$

Substituting a2, a1, a0, g, into Eq.(13) and setting all the coefficients of powers $X$ to be zero, then we obtain a system of nonlinear algebraic equations and by solving it, we obtain $\mathrm{A} 1=0 ; \mathrm{A} 0=0$ where $\mathrm{k} \neq 0, \lambda, 1$, and $\mathrm{p}$ are constants. Using the conditions $\mathrm{A} 1=0 ; \mathrm{A} 0=0$ and Eq.(9), we obtain the traveling wave solution of the Eq.(6)

$$
\left.u(x, y, z, t)=\frac{\lambda-3 l^{2}-3 p^{2}}{2}\left(1+\tan \phi^{2}\right)\right)
$$

where $\phi=\frac{\sqrt{3 l^{2}-\lambda+3 p^{2}}}{2}(x+l y+p z-\lambda t)$.

\section{Conclusions}

The travelling wave solutions for the Eq.(6) are obtained by using the first integral method. People can extract important information from the singularity when studying the water waves of long wavelength with weakly non-linear restoring forces and frequency dispersion and model waves in ferromagnetic media. In all, the first integral method is a standard, direct and computerizable method. The performance of this method is reliable and effective, especially for the nonlinear equation with complicated and tedious algebraic calculation.

\section{Acknowledgements}

This work was financially supported by the National Natural Science Foundation of China (Grant Nos 71573027, 11504283 and 11447115).

\section{References}

[1] T.C. Xia, B. Li, H.Q. Zhang, New explicit and exact solutions for the Nizhnik-Novikov-Vesselov equation, Appl. Math. ENotes 1 (2001) 139-142.

[2] Z. Sheng, The periodic wave solutions for the $(2+1)$-dimensional Konopelchenko-Dubrovsky equations, Chaos Solitons Fractals 30 (2006) 1213-1220.

[3] M. Inc, M. Ergut, Periodic wave solutions for the generalized shallow water wave equation by the improved Jacobi elliptic function method, Appl. Math. E-Notes 5 (2005) 89-96. 
[4] A. Bekir, The tanh-coth method combined with the Riccati equation for solving nonlinear equation. Chaos Soliton Fract, 40 (2009) 1467-1474.

[5] W. Malfliet, W. Hereman, The tanh method. I: Exact solutions of nonlinear evolution and wave equations. Physica Scripta 1996;54:563.

[6] AM. Wazwaz, The tanh method for travelling wave solutions of nonlinear equations. Appl Math Comput 2004;154(3):713.

[7] E. Yusufog, A. Bekir, Exact solutions of coupled nonlinear evolution equations. Chaos Soliton Fract 2008;37(3):842.

[8] Z. Feng, The first integer method to study the BurgersKorteweg-de Vries equation, J. Phys. A 35 (2) (2002) 343-349.

[9] Z. Feng, On explicit exact solutions to the compound BurgersKdV equation, Phys. Lett., A, 293(1-2), pp. 57-66, 2002.

[10] T.R. Ding, C.Z. Li, Ordinary Differential Equations, Peking University Press, Peking, 1996.

[11] H. Jafaria, A. Soorakia, Y. Talebia, A. Biswas, The first integral method and traveling wave solutions to Davey-Stewartson equation, Nonlinear Analysis: Modelling and Control, 2012, Vol. 17, No. 2, 182-193.

[12] N. Taghizadeh, M. Mirzazadeh, F. Farahrooz, Exact solutions of the nonlinear Schrodinger equation by the first integral method, J. Math. Anal. Appl. 374 (2011) 549-553.

[13] N. Taghizadeh, M. Mirzazadeh, F. Tascan, The first-integral method applied to the Eckhaus equation, Applied Mathematics Letters 25 (2012) 798-802.

[14] S. Abbasbandy, A. Shirzadi, The first integral method for mod-4 ified Benjamin-Bona-Mahony equation, Commun. Nonlinear Sci. Numer. Simul. 15 (2010) 1759-1764.

[15] A. Bekir, O. Guner, O. Unsal, The First Integral Method for Exact Solutions of Nonlinear Fractional Differential Equations, Journal of Computational and Nonlinear Dynamics MARCH 2015, Vol. 10, 021020-1.

[16] F. Tascan , A. Bekir, M. Koparan, Travelling wave solutions of nonlinear evolution equations by using the first integral method, Commun Nonlinear Sci Numer Simulat 14 (2009) 1810-1815.

[17] A. D. Polyanin and A. I. Zhurov, Exact Solutions to Some Classes of Nonlinear Integral, Integro-functional, and Integrodifferential Equations, MATHEMATICAL PHYSICS, 2008, Vol. 419, No. 1, pp. 30-34.

[18] W. Sapto, A.G. Ramm, arXiv:0911.3071v2.

[19] https://en.wikipedia.org/wiki/Kadomtsev-Petviashviliequation.

[20] F. Elsabbagh, A.T. Ali, New Exact Solutions for (3+1)-Dimensional Kadomtsev- Petviashvili Equation and Generalized (2+1)-Dimensional Boussinesq Equation, International Journal of Nonlinear Sciences Numerical Simulation , 2005 , 6 (2):151-162.

[21] M. Khalfallah, New exact traveling wave solutions of the $(3+1)$ dimensional KadomtsevPetviashvili (KP) equation, Communications in Nonlinear Science and Numerical Simulation, 2009, 14 (4) :1169-1175.

[22] F. Xie, Y. Zhang, Z. Lu, Symbolic computation in nonlinear evolution equation: application to (3+1)-dimensional Kadomtsev-Petviashvili equation, Chaos Solitons and Fractals, 2005, 24 (1):257-263 . 